\title{
New trends in the multidisciplinary treatment of liver tumors
}

\section{Marcello Donati*l \& Francesco Basilel}

'Department of Surgery. General \& Oncologic Surgery Unit. Vittorio-Emanuele University Hospital of Catania Via Plebiscito 628, 95122 Catania, Italy

*Author for correspondence: Tel. : +39 0957435053 = Fax: +39095 7435117 m mar_donati@libero.it

\section{3rd Interdisciplinary Treatment of Liver Tumors Congress}

\section{8-20 April 2013, Essen, Germany}

The authors report on new trends in the multidisciplinary treatment of liver tumors from the 3rd Interdisciplinary Treatment of Liver Tumors Congress in Essen, Germany. Novel aspects of molecular target-oriented therapies of hepatocarcinoma were reported, such as the current position of radiofrequency and microwave ablation of tumors compared with the new electroporation technique. State-of-the-art and new surgical trends in cholangiocarcinoma treatment were also discussed. The correlation of the biological behavior involved in the differentiation of melanomas with the treatment of liver metastasis was better defined. The role of neoadjuvant chemotherapy and combined strategies for colorectal liver metastasis were focused on intra-arterial therapeutic options. New data on neuroendocrine tumor liver metastases were also shared, leading to a better orientation for patient selection for surgical treatment.

Every 2 years an international congress dealing with the interdisciplinary treatment of liver tumors takes place in order to update oncologists, surgeons, hepatologists and, generally, all specialists engaged in liver tumor treatments in a multidisciplinary manner. This year the meeting took place in April in Essen (Germany). The event, organized by Wilke from the Department of Oncology of the University of Essen (Essen, Germany) was also supported by the European Society of Surgical Oncology and certified by the European Society of Medical Oncology. More than 50 renowned speakers took part in the meeting with approximately 400 participants attending the scientific program and satellite symposia organized by many exhibitors and sponsors. Many speakers were recognized experts in the field of oncology, surgery, interventional radiology and gastroenterology, and shared data and published information in an evidence-based manner with participants, resulting in long discussions together with panel experts and moderators. A poster area was also present sharing many international contributions in a multidisciplinary fashion with participants, presenting contributions from many groups from all over the world.

This short article intends to focus on the most important data, points of view or innovations reported in the main speeches during this very exciting meeting; it is divided into the principal sections of the meeting focused on the specific diseases that were discussed during the 3 days of the conference.

\section{Hepatocellular carcinoma}

One of the most interesting speeches was given by Carmeliet from the Vesalius Research Center at Katholieke Universiteit Leuven (Leuven, Belgium), a renowned researcher who has been engaged for many years in the challenging field of molecular aspects of vascular proliferation pathways in tumors. The new and effective chemotherapics (e.g., bevacizumab and cetuximab) have undergone testing and are now entered into clinical practice in combination with other drugs in the form of protocols intending to limit vascular proliferation and by acting on VEGF or EGFRs in order to arrest such an intense stimulation [1]. It must be emphasized that patients can very rapidly develop resistance to such drugs [2] and that many mechanisms are responsible for this. Carmeliet showed some models explaining the dynamics of vascular proliferation inside the tumor, which because of the anarchic structure of the vessels produces 'highly vascularized' areas as well as 'ischemic' ones, with the latter causing additional production of VEGF, which sustains this vicious circle of vascular proliferation. The aim of new target therapies is no longer to block VEGFRs (owing to referred resistance), but to act directly on periendothelial cells, inducing architectually 'regular' vascular proliferation rather than anarchic proliferation, in order to reduce the malignant spread of tumors therefore maintaining the benign biologic behavior of the tumor. The conversion to a benign type of vascular proliferation

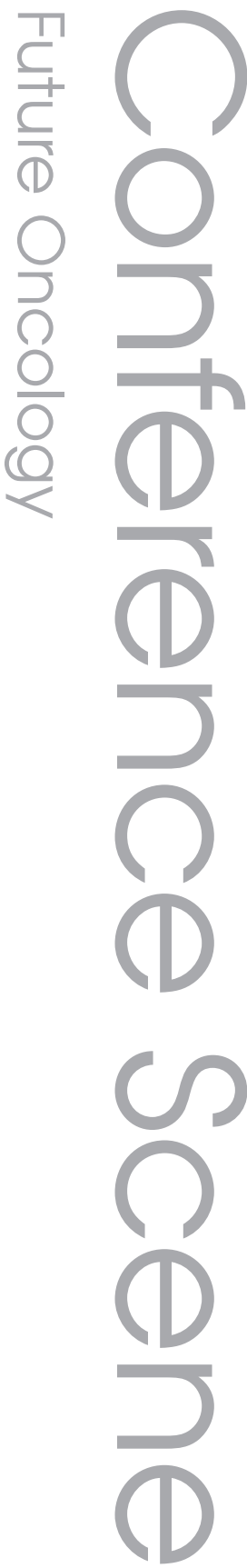

\section{Keywords}

- antiangiogenic agent

- hepatic arterial infusion

- in situ split - liver tumor

- multidisciplinary treatment

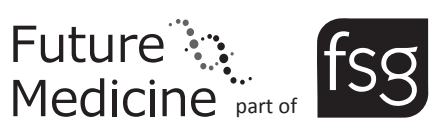


could avoid the vicious circle from ischemia to vascular proliferation factor induction, anarchic proliferation, areas with strong perfusion/areas without any perfusion and ischemia. This was obtained in experimental cellular models with the administration of colchicine. These data are experimental, limited and not yet published; however, they are promising. This revolutionary approach could open a window for new vascular target-oriented strategies in molecular oncology.

The size of the lesion also has a big impact on the effectiveness of treatments, particularly for radiofrequency ablation (RFA) and microwave ablation (MWA), for hepatocellular carcinoma, as was summarized by Crocetti from the University of Pisa (Pisa, Italy), who offered a good overview of the advantages of RFA compared with MWA. RFA appears to be effective for lesions up to $3 \mathrm{~cm}$ distant from the vessels due to the heat-sink effect (efficacy: 97\% and 5-year survival: 68\%) [3], while MWA seems to overcome size limitation of RFA treating lesions up to $7 \mathrm{~cm}$, showing a faster ablation procedure, a reduced heat-sink effect and improved convection profile; however, MWA seems to be more appropriate for superficial lesions. Randomized controlled trials, comparing both methods, showed no difference in efficacy, but showed a slightly lower recurrence and complication rate for RFA. MWA is, however, in its infancy. The new irreversible electroporation was presented as a new method allowing disruption of transmembrane potential and cell death without any need for pharmacological therapy. To date only data on preclinical models and very initial clinical experiences are available [4]. The advantages of this method seem to be: no size limitations; mantaining the integrity of vasculare structures involved in the tumor (destroying tumor cells but preserving large vessels); and no thermic effects on liver parenchyma. Clinical studies on this topic are required.

\section{Cholangiocarcinoma}

Lang, a surgeon from Mainz University (Mainz, Germany) reported an overview of state-of-theart cholangiocarcinoma surgical treatments with curative or palliative intent. As is known among specialists, the surgical treatment of cholangiocarcinoma especially the hilar type (so called 'Klatskin tumors') is one of the most engaging surgical fields [5]. Reporting the experience of the Mainz group, it was clear that preoperative diagnosis still has significant limitations, and resectability criteria are mainly based on a surgeon's experience and ability. The Mainz group reported that approximately $76 \%$ of cholangiocarcinomas were judged surgically resectable, but from the histologic response $85 \%$ of them were R0 resected, this should be considered a common problem for surgeons engaged in this type of surgery. The surgical tactics to achieve R0 can vary from extended hepatectomies to mesohepatectomies. Lang also reported the new interesting application of associating liver partition with portal vein ligation for the staged hepatectomy technique to some patients as a newly available technical tool with the potential to extend resectability criteria of Klatskin's tumors [6]. The speaker also underlined the poor prognosis of this group of patients with Klatskin's tumors, despite the great surgical research efforts of previous years.

\section{Melanoma}

Keihlolz from the Charitè Comprehensive Cancer Center (Berlin, Germany) reported in his talk the important differences between the four melanomas (cutaneous solar, cutaneous nonsolar, mucosal and uveal). This differentiation is currently of capital importance as it has become the cornerstone for target-oriented differentiation of oncological treatments. In fact, among those four types of melanoma, many differences in genomic mutations (braf, met and c-kit) reflect different biological behaviors and, therefore, different sensitivities to molecular therapies [7].

We can also interpret the talk of Adam from the Paul Brousse Hospital (Vilejuif, France) reporting on how this differentiation has a clinical consequence especially for the treatment of metastatic liver disease along similar lines. In fact the largest series published shows how some selection criteria for treatment of metastatic liver melanomas should be used in order to improve clinical outcomes. Liver metastases from uveal melanomas seem to have a significantly better prognosis, which, to date, seems to be the only type of melanoma in which liver resection improves prognosis [8], mainly in patients with one to three metastases and a metachronous presentation of at least 2 years disease-free survival. In selected cases rehepatectomy could also be taken into consideration. Liver metastatic cutaneous melanomas recur usually very early on and frequently. Present diagnostic options does not allow to detect miliaric dissemination of melanomas (typical metastatic pattern of this tumor); to date this remains an unsolved problem.

\section{Colorectal liver metastases}

Among the highlights of this section the talk by Kemeny from the Memorial Sloan-Kettering 
Cancer Center (NY, USA) has to be mentioned. She focused the auditorium's attention on the efficacy of chemotherapy in colorectal liver metastases treatment. The trend seems to be a chemotherapy-first approach, not only for the initially irresectable lesions but also for resectable ones, since chemotherapy (folinic acid, 5-fluorouracil and oxaliplatin, and folinic acid, 5-fluorouracil and irinotecan) has shown a significantly better prognosis in combination with liver resection for this cohort of patients [9]. The speaker also underlined the important role of hepatic arterial infusion (HAI), which is increasingly used among liver centers. Four large randomized studies have clearly shown advantages in recurrence-free survival for HAI compared with systemic chemotherapy. New trials conducted at the Memorial Sloan-Kettering Cancer Center on more than 100 patients are showing a 4-year survival of approximately $80 \%$ after liver resection combined with HAI and systemic therapy.

\section{Neuroendocrine tumor metastases}

The speech of Pawlik from Johns Hopkins Hospital (MD, USA) questioned the role of intra-arterial therapy for neuroendocrine tumor metastases instead of surgery [10]. Approximately $40 \%$ of patients affected by neuroendocrine tumors will develop metastases. A stratification of patients following clinical presentation and grade of liver involvement has shown that patients with little parenchymal involvement $(<25 \%)$, even if asymptomatic and symptomatic patients with massive liver involvement show the best long-term outcomes, while asymptomatic patients with massive involvement of the liver show no benefit from liver resection owing to very early relapse. In this cohort of patients intrarterial therapies, such as transarterial chemoembolization, transarterial embolization, drug-eluting bead therapy or yttrium-90 microspheres radioembolization therapy, are intended to be the best therapeutic options to achieve maximum advantages. Persistent recurrence for metacrone metastases with a long disease-free interval was explained by entire microscopic analysis of all resected specimens, which showed, for this kind of tumor, a microscopic form of miliaric dissemination together with clinically evident and computer topography-detectable liver metastases.

\section{Conclusion}

Taking into account the growing importance of co-operation among specialists of different areas, resulting in certified tumor boards in many centers, these kinds of meetings will, in the future, allow knowledge exchange and collaboration in the complex scenario of liver tumors. The great progress of oncology over the last few years is now permitting us to treat patients with advanced diseases, who, in the past, were considered unsuitable for surgery or even any kind of treatment. The correct differentiation of indications, field of therapeutic interventions and sequential multidisciplinary therapy will better define the correct evidence-based fields of action of each specialist area through the ever better stratification of patients. The progressive evolution of molecular oncology is today allowing a better stratification of patients in order to identify subcohorts that could benefit from specific treatment protocols. We should be grateful to the organizers and sponsors for their efforts in gathering such an interesting panel of experts from all over the world, to share knowledge, trends and perspectives on a multidisciplinary approach to the treatment of liver tumors.

\section{Financial \& competing interests disclosure \\ The authors have no relevant affiliations or financial involvement with any organization or entity with a financial interest in or financial conflict with the sub- ject matter or materials discussed in the manuscript. This includes employment, consultancies, honoraria, stock ownership or options, expert testimony, grants or patents received or pending, or royalties. \\ No writing assistance was utilized in the production of this manuscript.}

\section{References}

1. Zhu AX. New agents on the horizon in hepatocellular carcinoma. Ther. Adv. Med. Oncol. 5(1), 41-50 (2013).

2. Fang P, Hu JH, Cheng ZG, Liu ZF, Wang JL, Jiao SC. Efficacy and safety of bevacizumab for the treatment of advanced hepatocellular carcinoma: a systematic review of Phase II trials. PloS One 7(12), e49717 (2012).
3. Lencioni R, Crocetti L. Local-regional treatment of hepatocellular carcinoma. Radiology 262(1), 43-58 (2012).

4. Cheung W, Kavnoudias H, Roberts S, Szkandera B, Kemp W, Thomson KR. Irreversible electroporation for unresectable hepatocellular carcinoma: initial experience and review of safety and outcomes. Technol. Cancer Res. Treat. 12(3), 233-241 (2013).
5. Donati M, Basile F. Resectability criteria for Klatskin tumours: the "black run" of liver surgery. J. Solid Tumors 2(4), 1-3 (2012).

6. Donati M, Stavrou G, Basile F, Gruttadauria S, Niehaus KJ, Oldhafer KJ. Combination of in situ split and portal ligation: lights and shadows of a new surgical procedure. Ann. Surg. 256(3), e11-e12 (2012). 
7. Woodman SE. Metastatic uveal melanoma: biology and emerging treatments. Cancer J. 18(2), 148-152 (2012).

8. Mariani P, Piperno-Neumann S, Servois V et al. Surgical management of liver metastases from uveal melanoma: 16 years' experience at the Institut Curie.
Eur. J. Surg. Oncol. 35(11), 1192-1197 (2009).

9. Oh SY, Kim do Y, Kim YB, Suh KW. Comparison of oncological outcomes between neoadjuvant and adjuvant chemotherapy combined with surgery for resectable synchronous colorectal liver metastases. J. Surg. Res. 182(2), 257-263 (2013).

10. Arrese D, McNally ME, Chokshi R et al. Extrahepatic disease should not preclude transarterial chemoembolization for metastatic neuroendocrine carcinoma. Ann. Surg. Oncol. 20(4), 1114-1120 (2013). 